\title{
Endoscopic features of primary amyloidosis of the stomach
}
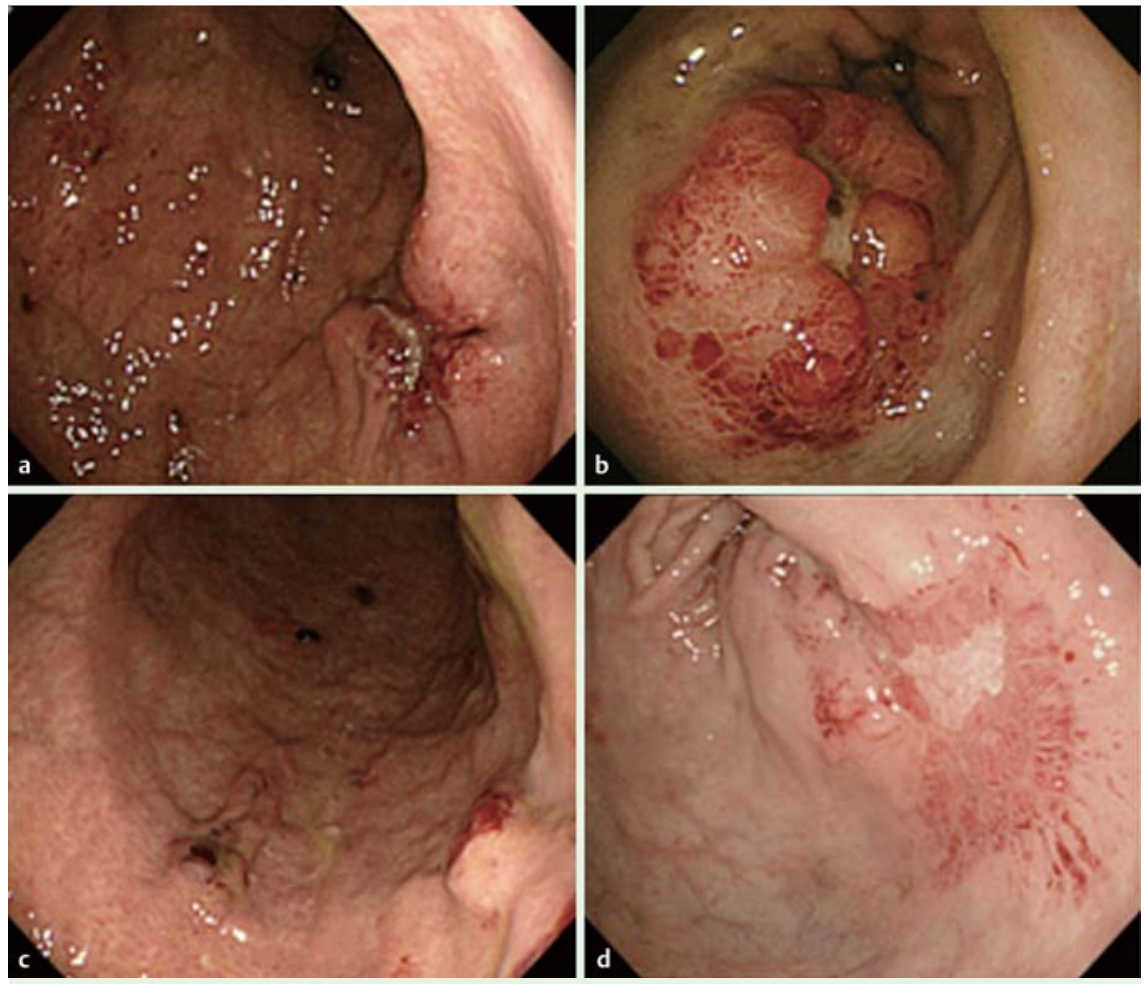

Fig. 1 Gastroscopy (white-light examination) in a 72-year-old-woman with a history of amyloidosis. a An ulcerative lesion resembling advanced gastric cancer in the proximal stomach. $\mathbf{b}$ An elevated lesion with ulcer, similar to the submucosal tumor in the antrum. $\mathbf{c}$ Intramural hematomas and flat elevations in the middle stomach. $\mathbf{d}$ A small, shallow ulcer surrounded by erythema in the middle stomach. The presence of residual food suggested gastroparesis.

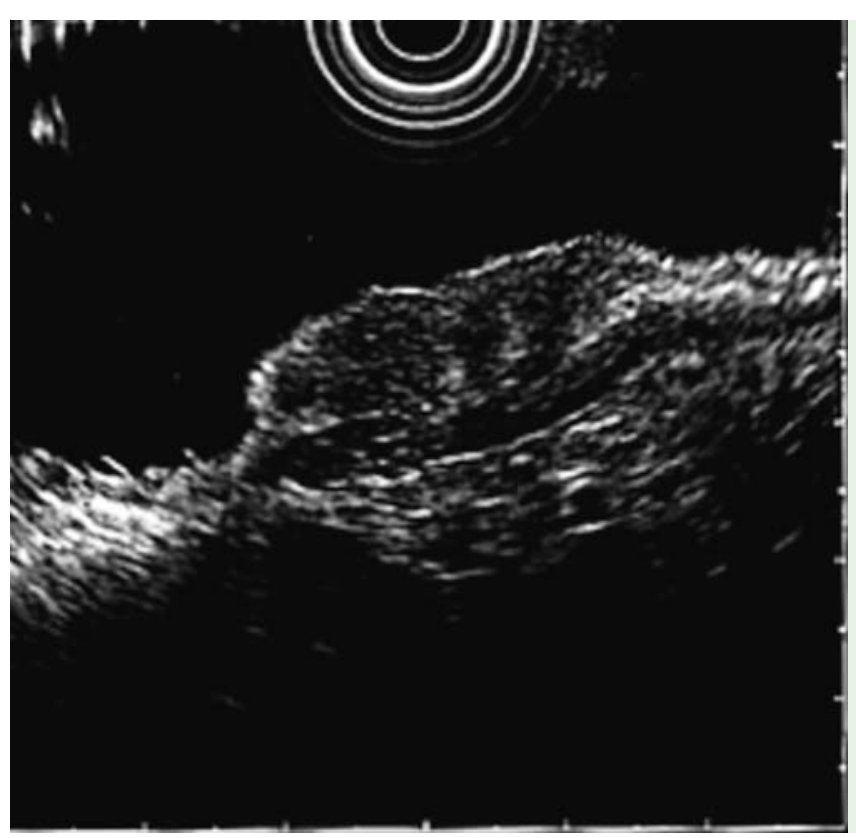

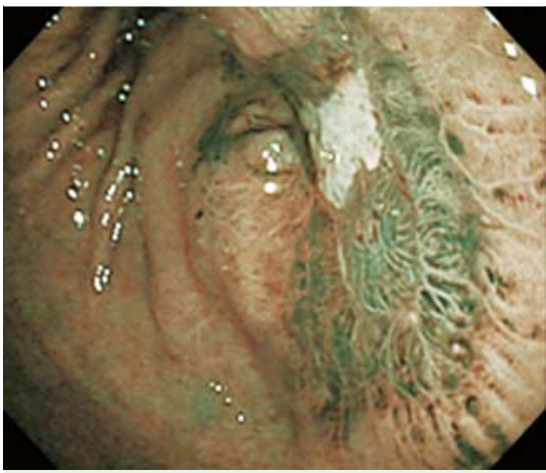

Fig. 2 Narrow-band imaging (NBI) view of the same lesion of $\bullet$ Fig. $1 \mathrm{~d}$, showing grayishgreen mucosa surrounding a small ulcer, without an obvious vascular pattern.

A 72-year-old-woman, with a history of resection of tracheal $(\mathrm{AL})$ amyloidosis at age 61 and biopsy-based diagnosis of gastric amyloidosis at age 68, was admitted to our hospital. No amyloid deposits had been detected in any other organ and multiple myeloma had been excluded. Gastroscopy showed several findings, including ulcerative lesions mimicking advanced cancer, submucosal tumor-like lesions, intramural hematomas, small ulcers, and flat elevated lesions ( $\bullet$ Fig. 1 ). With narrow-band imaging (NBI) enhancement, the mucosa surrounding the ulcer appeared grayish-green in color ( Fig.2). Endoscopic ultrasound (EUS) revealed hypoechoic thickening of the mucosa and submucosa, with the loss of the normal sonographic layers ( Fig.3). Histological examination with Congo red staining revealed amorphous eosinophilic infiltrates, which also showed positive immunostaining for both kappa and lambda light chains, but not amyloid $\mathrm{A}$, validating the diagnosis of gastric primary (AL) amyloidosis. Endoscopic examination and biopsy samples ruled out lesions in other gastrointestinal locations. However, there was massive bleeding on biopsy of the ulcerative lesion and blood transfusion was required.

Endoscopic findings in gastric amyloidosis are nonspecific, including a variety of features such as erosions, fine granular- 
appearing mucosa, mucosal friability, enlarged folds, hematoma, polyps, ulcers, and submucosal tumor-like lesions $[1,2]$. Regarding EUS findings in gastric amyloidosis, thickening of mucosa and submucosa and loss of the normal layered structure have been reported $[3,4]$. EUS may be helpful for assessing the extent and areas of amyloid deposition, especially in cases presenting with prominent folds and/or a poorly distensible stomach with normal-appearing mucosa [3]. Recently, the usefulness of NBI for diagnosing rectal amyloidosis has been highlighted [5]. In the present case, the erythematous area surrounding the ulcer also appeared grayish-green in color with NBI, indicating clinical applicability of the method in gastric amyloidosis. Although it is sometimes difficult to distinguish amyloid lesions from malignancies, both EUS and NBI may help avoid biopsies with their attendant risk of hemorrhage as was seen in our case.
Endoscopy_UCTN_Code_CCL_1AB_2AD_3AF

Competing interests: None

\section{T. Sawada', Y. Adachi', 2, ${ }^{1}$ K. Akino ${ }^{1}$,} Y. Arimura², T. Ishida², Y. Ishii ${ }^{3}$, T. Endo ${ }^{1}$

${ }^{1}$ Division of Gastroenterology, Department of Internal Medicine, Sapporo

Shirakaba-dai Hospital, Sapporo, Japan

2 First Department of Internal Medicine, Sapporo Medical University, Sapporo, Japan

${ }^{3}$ Department of Pathology, Sapporo Shirakaba-dai Hospital, Sapporo, Japan

\section{References}

1 Ebert EC, Nagar M. Gastrointestinal manifestations of amyloidosis. Am J Gastroenterol 2008; 103: 776-787

2 Tada S, Iida M, Iwashita A. Endoscopic and biopsy findings of the upper digestive tract in patients with amyloidosis. Gastrointest Endosc 1990; 36: 10-14

3 Grape T, Wurm Johansson G, Eriksson M et al. Primary gastroduodenal amyloidosis. Endoscopy 2011; 43: E288
4 Gandolfi L, Colecchia A, Leo P et al. Endoscopic ultrasonography in the diagnosis of gastrointestinal amyloid deposits: clinical case report. Endoscopy 1995; 27: 132-134

5 Hui YT, Lam TW, Yee Lam PW et al. Narrowband imaging system with magnifying endoscopy for rectal amyloidosis. Gastrointest Endosc 2008; 68: 400-401

\section{Bibliography}

Dol http://dx.doi.org/

10.1055/s-0032-1309750

Endoscopy 2012; 44: E275-E276

(c) Georg Thieme Verlag KG

Stuttgart · New York

ISSN 0013-726X

\section{Corresponding author}

\section{Y. Adachi}

Division of Gastroenterology

Department of Internal Medicine

Sapporo Shirakaba-dai Hospital

2-18 Tsukisamu-Higashi, Toyohira-Ku

Sapporo 062-0052

Japan

Fax: +81-11-852-8194

yadachi@sapmed.ac.jp 